\title{
SEPSIS MORTALITY SCORE USING MULTI-MARKER APPROACH FOR THE PREDICTION OF MORTALITY IN SEPTIC CRITICALLY ILL PATIENTS
}

\author{
Wan Fadzlina Wan Muhd Shukeri ${ }^{1}$, Azrina Md. Ralib ${ }^{1}$ and Mohd Basri Mat-Nor ${ }^{1}$ \\ ${ }^{1}$ Department of Anesthesiology and Critical Care, Kuliyyah of Medicine, International \\ Islamic University of Malaysia
}

Presenter: Wan Fadzlina Wan Muhd Shukeri, wfwms82@yahoo.co.uk

Introduction: Clinical scoring methods such as the Sequential Organ Failure Assessment (SOFA) score are frequently used to predict outcome in sepsis, with limited capacity. Further tools for risk assessment of septic critically ill patients would thus be useful. Our purpose was to derive a scoring method i.e. Sepsis Mortality Score using multimarker approach for the prediction of 30-day mortality in septic critically ill patients, and compare its performance to the SOFA score.

Materials and method: This prospective study included 159 septic patients admitted to an intensive care unit (ICU). Leukocytes count, procalcitonin (PCT), interleukin-6 (IL-6), paraoxonase (PON) and arylesterase (ARE) were assayed from blood draws obtained on ICU presentation. Logistic regression was used to identify an optimal combination of these biomarkers to create the Sepsis Mortality Score, which was the predicted probability of 30-day mortality.

Results: The 30-day mortality rate was $28.9 \%$. In the logistic regression model, leukocytes count, PCT, IL- 6 and ARE remained as independent biomarkers. The areas under curve (AUC) of the Sepsis Mortality Score derived from these biomarkers was 0.814 ( $95 \%$ confidence interval $(\mathrm{Cl})$ 0.736-0.892), better than the SOFA score (AUC 0.767 (95\% $\mathrm{Cl}$ 0.677-0.857). Addition of Sepsis Mortality Score on SOFA score showed an even better prediction of 30-day mortality (AUC 0.845 (95\% Cl 0.772-0.919)).

Conclusion: Multi-marker approach using leukocytes count, PCT, IL-6 and ARE, combined with the SOFA score, might provide a valuable tool for risk assessment of septic critically ill patients. 